Supplement of Biogeosciences, 11, 4029-4038, 2014

http://www.biogeosciences.net/11/4029/2014/

doi:10.5194/bg-11-4029-2014-supplement

(C) Author(s) 2014. CC Attribution 3.0 License.

(c) (i)

Supplement of

\title{
Experimental evidence for foraminiferal calcification under anoxia
}

M. P. Nardelli et al.

Correspondence to: M. P. Nardelli (mariapia.nardelli@univ-angers.fr) 


\section{Supplement}

Table S1. Summary of main results obtained for Experiment 1: Ammonia tepida. Both values obtained for each single replicate and averages are shown.

\begin{tabular}{|c|c|c|c|c|c|c|c|c|c|c|c|}
\hline Replicate & Layer & $\begin{array}{l}\text { Depth } \\
(\mathrm{cm})\end{array}$ & $\begin{array}{c}\text { Initial } \\
\text { number } \\
\text { of } \\
\text { specimens }\end{array}$ & $\begin{array}{c}\text { Number of } \\
\text { re-found } \\
\text { specimens }\end{array}$ & $\begin{array}{c}\text { Living } \\
\text { (n) }\end{array}$ & $\begin{array}{c}\text { Dead } \\
\text { (n) }\end{array}$ & $\begin{array}{c}\text { Living } \\
(\%)\end{array}$ & $\begin{array}{c}\text { Dead } \\
(\%)\end{array}$ & $\begin{array}{c}\text { Specimens } \\
\text { with new } \\
\text { chambers (n) }\end{array}$ & & \\
\hline \multirow[t]{7}{*}{$\mathrm{R} 1$} & 1 & $0-0.3$ & 50 & 45 & 14 & 31 & 31.11 & 68.89 & 0 & & \\
\hline & 2 & $0.3-1.3$ & 50 & 43 & 32 & 11 & 74.42 & 25.58 & 0 & & \\
\hline & 3 & $1.3-2.3$ & 49 & 43 & 21 & 22 & 48.84 & 51.16 & 0 & & \\
\hline & 4 & $2.3-3.3$ & 50 & 36 & 29 & 7 & 80.56 & 19.44 & 0 & & \\
\hline & 5 & $3.3-4.3$ & 50 & 44 & 27 & 17 & 61.36 & 38.64 & 0 & & \\
\hline & 6 & $4.3-7.3$ & 49 & 40 & 32 & 8 & 80.00 & 20.00 & 3 & & \\
\hline & 7 & $7.3-10.3$ & 45 & 35 & 27 & 8 & 77.14 & 22.86 & 0 & & \\
\hline \multirow[t]{7}{*}{$\mathrm{R} 2$} & 1 & \begin{tabular}{|c|}
$0-0.3$ \\
\end{tabular} & 50 & 41 & 21 & 20 & 51.22 & 48.78 & 2 & & \\
\hline & 2 & $0.3-1.3$ & 44 & 38 & 14 & 24 & 36.84 & 63.16 & 0 & & \\
\hline & 3 & $1.3-2.3$ & 46 & 43 & 39 & 4 & 90.70 & 9.30 & 0 & & \\
\hline & 4 & $2.3-3.3$ & 45 & 35 & 31 & 4 & 88.57 & 11.43 & 0 & & \\
\hline & 5 & $3.3-4.3$ & 48 & 31 & 18 & 13 & 58.06 & 41.94 & 0 & & \\
\hline & 6 & 4.3-7.3 & 49 & 35 & 27 & 8 & 77.14 & 22.86 & 0 & & \\
\hline & 7 & 7.3-10.3 & 49 & 43 & 23 & 20 & 53.49 & 46.51 & 1 & & \\
\hline \multirow[t]{7}{*}{ R3 } & 1 & \begin{tabular}{|c|}
$0-0.3$ \\
\end{tabular} & 49 & 43 & 27 & 16 & 62.79 & 37.21 & 1 & & \\
\hline & 2 & $0.3-1.3$ & 50 & 40 & 34 & 6 & 85.00 & 15.00 & 0 & & \\
\hline & 3 & $1.3-2.3$ & 50 & 43 & 25 & 18 & 58.14 & 41.86 & 2 & & \\
\hline & 4 & $2.3-3.3$ & 50 & 12 & 12 & 0 & 100.00 & 0.00 & 1 & & \\
\hline & 5 & $3.3-4.3$ & 50 & 41 & 34 & 7 & 82.93 & 17.07 & 5 & & \\
\hline & 6 & $4.3-7.3$ & 46 & 35 & 27 & 8 & 77.14 & 22.86 & 0 & & \\
\hline & 7 & 7.3-10.3 & 48 & 38 & 26 & 12 & 68.42 & 31.58 & 0 & & \\
\hline \multirow[t]{8}{*}{ Average } & Layer & $\begin{array}{c}\text { Depth } \\
(\mathrm{cm})\end{array}$ & $\begin{array}{l}\text { Average } \\
\text { living }(\mathrm{n})\end{array}$ & $\begin{array}{c}\text { s.d. } \\
\text { mean }\end{array}$ & $\begin{array}{l}\text { Average } \\
\text { dead (n) }\end{array}$ & $\begin{array}{l}\text { s.d. } \\
\text { mean }\end{array}$ & \begin{tabular}{|c|} 
Average \\
living (\%)
\end{tabular} & $\begin{array}{c}\text { s.d. } \\
\text { mean }\end{array}$ & $\begin{array}{c}\text { Specimens } \\
\text { with new } \\
\text { chambers } \\
(\%)\end{array}$ & $\begin{array}{l}\text { s.d. } \\
\text { mean }\end{array}$ & $\begin{array}{c}\text { Total } \\
\text { new } \\
\text { chambers } \\
\text { (n) }\end{array}$ \\
\hline & 1 & $0-0.3$ & 20.7 & 6.5 & 22.3 & 7.8 & 48.37 & 9.26 & 2.40 & 1.41 & 3 \\
\hline & 2 & $0.3-1.3$ & 26.7 & 11.0 & 13.7 & 9.3 & 65.42 & 14.61 & 0.00 & 0.00 & 0 \\
\hline & 3 & $1.3-2.3$ & 28.3 & 9.5 & 14.7 & 9.5 & 65.89 & 12.69 & 1.55 & 1.55 & 2 \\
\hline & 4 & $2.3-3.3$ & 24.0 & 10.4 & 3.7 & 3.5 & 89.71 & 5.64 & 2.78 & 2.78 & 1 \\
\hline & 5 & $3.3-4.3$ & 26.3 & 8.0 & 12.3 & 5.0 & 67.45 & 7.80 & 4.07 & 4.07 & 5 \\
\hline & 6 & $4.3-7.3$ & 28.7 & 2.9 & 8.0 & 0.0 & 78.10 & 0.95 & 2.50 & 2.50 & 3 \\
\hline & 7 & 7.3-10.3 & 25.3 & 2.1 & 13.3 & 6.1 & 66.35 & 6.91 & 0.78 & 0.78 & 1 \\
\hline
\end{tabular}


Table S2. Summary of main results obtained for Experiment 2: Bulimina marginata. Both values obtained for each single replicate and averages are shown.

\begin{tabular}{|c|c|c|c|c|c|c|c|c|c|c|c|}
\hline Replicate & Layer & $\begin{array}{l}\text { Depth } \\
(\mathrm{cm})\end{array}$ & $\begin{array}{l}\text { Initial } \\
\text { number } \\
\text { of } \\
\text { specimen } \\
\text { s }\end{array}$ & $\begin{array}{c}\text { Number } \\
\text { of re- } \\
\text { found } \\
\text { specimen } \\
\mathrm{s}\end{array}$ & $\begin{array}{l}\text { Living } \\
\text { (n) }\end{array}$ & $\begin{array}{c}\text { Dead } \\
\text { (n) }\end{array}$ & $\begin{array}{l}\text { Living } \\
(\%)\end{array}$ & $\begin{array}{c}\text { Dead } \\
(\%)\end{array}$ & $\begin{array}{c}\text { Specimens } \\
\text { with new } \\
\text { chambers } \\
\text { (n) }\end{array}$ & $\begin{array}{c}\text { Specimens } \\
\text { with new } \\
\text { chambers } \\
(\%)\end{array}$ & $\begin{array}{c}\text { Total new } \\
\text { chambers } \\
\text { (n) }\end{array}$ \\
\hline \multirow[t]{5}{*}{ R1 } & 1 & $0-0.3$ & 31 & 30 & 5 & 25 & 16.67 & 83.33 & 8 & 26.67 & 8 \\
\hline & 2 & $0.3-1.3$ & 31 & 29 & 8 & 21 & 27.59 & 72.41 & 9 & 31.03 & 9 \\
\hline & 3 & $1.3-2.3$ & 32 & 32 & 0 & 32 & 0.00 & 100.00 & 4 & 12.50 & 4 \\
\hline & 4 & $2.3-3.3$ & 31 & 21 & 1 & 20 & 4.76 & 95.24 & 0 & 0.00 & 0 \\
\hline & 5 & $3.3-4.3$ & 32 & 21 & 6 & 15 & 28.57 & 71.43 & 0 & 0.00 & 0 \\
\hline \multirow[t]{5}{*}{$\mathrm{R} 2$} & 1 & $0-0.3$ & 32 & 32 & 5 & 27 & 15.63 & 84.38 & 6 & 18.75 & 6 \\
\hline & 2 & $0.3-1.3$ & 32 & 31 & 12 & 19 & 38.71 & 61.29 & 6 & 19.35 & 8 \\
\hline & 3 & $1.3-2.3$ & 32 & 28 & 10 & 18 & 35.71 & 64.29 & 7 & 25.00 & 7 \\
\hline & 4 & $2.3-3.3$ & 31 & 29 & 4 & 25 & 13.79 & 86.21 & 4 & 13.79 & 4 \\
\hline & 5 & 3.3-4.3 & 31 & 28 & 7 & 21 & 25.00 & 75.00 & 0 & 0.00 & 0 \\
\hline \multirow[t]{5}{*}{ R3 } & 1 & $0-0.3$ & 32 & 31 & 8 & 23 & 25.81 & 74.19 & 15 & 48.39 & 18 \\
\hline & 2 & $0.3-1.3$ & 31 & 31 & 11 & 20 & 35.48 & 64.52 & 7 & 22.58 & 7 \\
\hline & 3 & $1.3-2.3$ & 32 & 32 & 15 & 17 & 46.88 & 53.13 & 8 & 25.00 & 8 \\
\hline & 4 & $2.3-3.3$ & 31 & 27 & 1 & 26 & 3.70 & 96.30 & 3 & 11.11 & 3 \\
\hline & 5 & $3.3-4.3$ & 31 & 30 & 9 & 21 & 30.00 & 70.00 & 0 & 0.00 & 0 \\
\hline \multirow[t]{6}{*}{ Average } & Layer & $\begin{array}{l}\text { Depth } \\
(\mathrm{cm})\end{array}$ & $\begin{array}{c}\text { Average } \\
\text { living } \\
\text { (n) }\end{array}$ & s.d. mean & $\begin{array}{c}\text { Average } \\
\text { dead } \\
\text { (n) }\end{array}$ & s.d. mean & $\begin{array}{c}\text { Average } \\
\text { living } \\
(\%)\end{array}$ & s.d. mean & $\begin{array}{c}\text { Specimens } \\
\text { with new } \\
\text { chambers } \\
(\%)\end{array}$ & s.d. mean & $\begin{array}{c}\text { Total new } \\
\text { chambers } \\
\text { (n) }\end{array}$ \\
\hline & 1 & $0-0.3$ & 6.5 & 2.1 & 24.0 & 1.4 & 19.37 & 3.23 & 31.27 & 8.86 & 29 \\
\hline & 2 & $0.3-1.3$ & 10.3 & 2.1 & 20.0 & 1.0 & 33.93 & 3.30 & 24.32 & 3.48 & 22 \\
\hline & 3 & $1.3-2.3$ & 8.3 & 7.6 & 22.3 & 8.4 & 27.53 & 14.14 & 20.83 & 4.17 & 19 \\
\hline & 4 & $2.3-3.3$ & 2.0 & 1.7 & 23.7 & 3.2 & 8.75 & 4.12 & 8.30 & 4.22 & 7 \\
\hline & 5 & $3.3-4.3$ & 7.3 & 1.5 & 19.0 & 3.5 & 27.50 & 2.04 & 0.00 & 0.00 & 0 \\
\hline
\end{tabular}


Table S3. Summary of main results obtained for Experiment 2: Cassidulina laevigata. Both values obtained for each single replicate and averages are shown. The specimens of this species were only introduced at layers 1, 2 and 4 of each replicate core.

\begin{tabular}{|c|c|c|c|c|c|c|c|c|c|c|c|}
\hline Replicate & Layer & $\begin{array}{l}\text { Depth } \\
(\mathrm{cm})\end{array}$ & $\begin{array}{c}\text { Initial } \\
\text { number of } \\
\text { specimens }\end{array}$ & $\begin{array}{c}\text { Number of } \\
\text { re-found } \\
\text { specimens }\end{array}$ & $\begin{array}{c}\text { Living } \\
\text { (n) }\end{array}$ & $\begin{array}{c}\text { Dead } \\
\text { (n) }\end{array}$ & $\begin{array}{c}\text { Living } \\
(\%)\end{array}$ & $\begin{array}{c}\text { Dead } \\
(\%)\end{array}$ & $\begin{array}{c}\text { Specimens } \\
\text { with new } \\
\text { chambers } \\
\text { (n) }\end{array}$ & $\begin{array}{l}\text { Specimens } \\
\text { with new } \\
\text { chambers } \\
(\%)\end{array}$ & $\begin{array}{c}\text { Total } \\
\text { new } \\
\text { chambers } \\
\text { (n) }\end{array}$ \\
\hline \multirow[t]{5}{*}{$\mathrm{R} 1$} & 1 & $0-0.3$ & 32 & 32 & 9 & 23 & 28.1 & 71.9 & 11 & 34.4 & 11 \\
\hline & 2 & $0.3-1.3$ & 33 & 30 & 9 & 21 & 30.0 & 70.0 & 7 & 23.3 & 7 \\
\hline & 3 & $1.3-2.3$ & - & - & - & - & - & - & - & - & - \\
\hline & 4 & $2.3-3.3$ & 33 & 32 & 0 & 32 & 0.0 & 100.0 & 6 & 18.8 & 6 \\
\hline & 5 & $3.3-4.3$ & - & - & - & - & - & - & - & - & - \\
\hline \multirow[t]{5}{*}{$\mathrm{R} 2$} & 1 & $0-0.3$ & 33 & 31 & 14 & 17 & 45.2 & 54.8 & 6 & 18.8 & 6 \\
\hline & 2 & $0.3-1.3$ & 32 & 30 & 8 & 22 & 26.7 & 73.3 & 6 & 20.0 & 6 \\
\hline & 3 & $1.3-2.3$ & - & - & - & - & - & - & - & - & - \\
\hline & 4 & $2.3-3.3$ & 32 & 28 & 0 & 28 & 0.0 & 100.0 & 4 & 14.3 & 4 \\
\hline & 5 & $3.3-4.3$ & - & - & - & - & - & - & - & - & - \\
\hline \multirow[t]{5}{*}{ R3 } & 1 & $0-0.3$ & 32 & 32 & 10 & 22 & 31.3 & 68.8 & 15 & 46.9 & 15 \\
\hline & 2 & $0.3-1.3$ & 33 & 28 & 6 & 22 & 21.4 & 78.6 & 7 & 25.0 & 7 \\
\hline & 3 & $1.3-2.3$ & - & - & - & - & - & - & - & - & - \\
\hline & 4 & $2.3-3.3$ & 32 & 32 & 0 & 32 & 0.0 & 100.0 & 5 & 15.6 & 5 \\
\hline & 5 & $3.3-4.3$ & - & - & - & - & - & - & - & - & - \\
\hline \multirow[t]{6}{*}{ Average } & Layer & $\begin{array}{l}\text { Depth } \\
(\mathrm{cm})\end{array}$ & $\begin{array}{l}\text { Average } \\
\text { living } \\
\text { (n) }\end{array}$ & $\begin{array}{l}\text { s.d. } \\
\text { mean }\end{array}$ & $\begin{array}{c}\text { Average } \\
\text { dead } \\
\text { (n) }\end{array}$ & $\begin{array}{l}\text { s.d. } \\
\text { mean }\end{array}$ & $\begin{array}{c}\text { Average } \\
\text { living } \\
(\%)\end{array}$ & $\begin{array}{c}\text { s.d. } \\
\text { mean }\end{array}$ & $\begin{array}{c}\text { Specimens } \\
\text { with new } \\
\text { chambers } \\
(\%)\end{array}$ & $\begin{array}{l}\text { s.d. } \\
\text { mean }\end{array}$ & $\begin{array}{c}\text { Total } \\
\text { new } \\
\text { chambers } \\
\text { (n) }\end{array}$ \\
\hline & 1 & $0-0.3$ & 11.0 & 2.6 & 34.8 & 3.2 & 34.8 & 5.2 & 33.3 & 8.14 & 32 \\
\hline & 2 & $0.3-1.3$ & 7.7 & 1.5 & 21.7 & 0.6 & 26.0 & 2.5 & 22.8 & 1.47 & 20 \\
\hline & 3 & $1.3-2.3$ & - & - & - & - & - & - & - & - & - \\
\hline & 4 & $2.3-3.3$ & 0.0 & 0.0 & 30.7 & 2.3 & 0.0 & 0.0 & 16.2 & 1.32 & 15 \\
\hline & 5 & $3.3-4.3$ & - & - & - & - & - & - & - & - & - \\
\hline
\end{tabular}


Figure S1. Element profiles for geochemical cores from Experiment 1: A. tepida

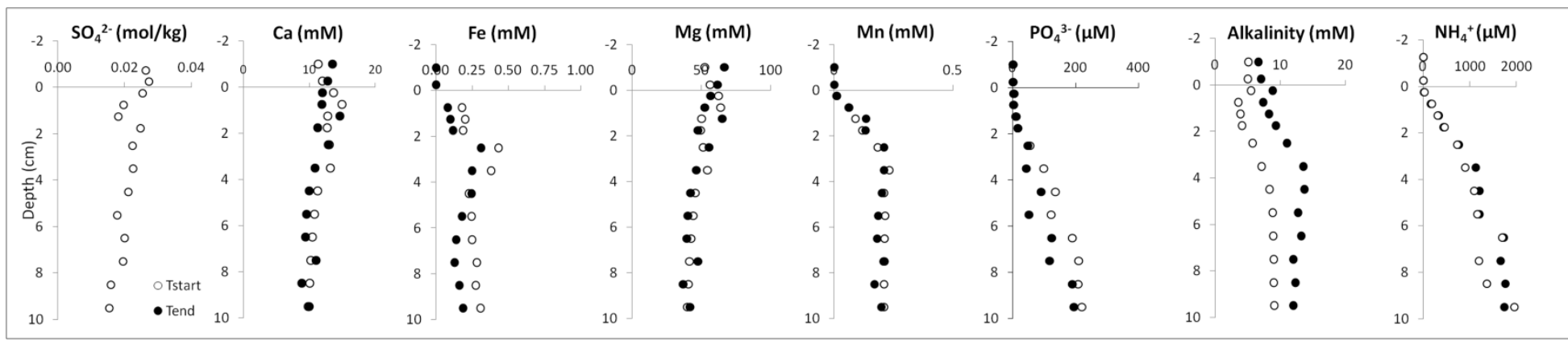

Figure S2. Element profiles for geochemical cores from experiment 2: B. marginata and C. laevigata. Please note that the scales for manganese, phosphates and ammonium are different from figure A1

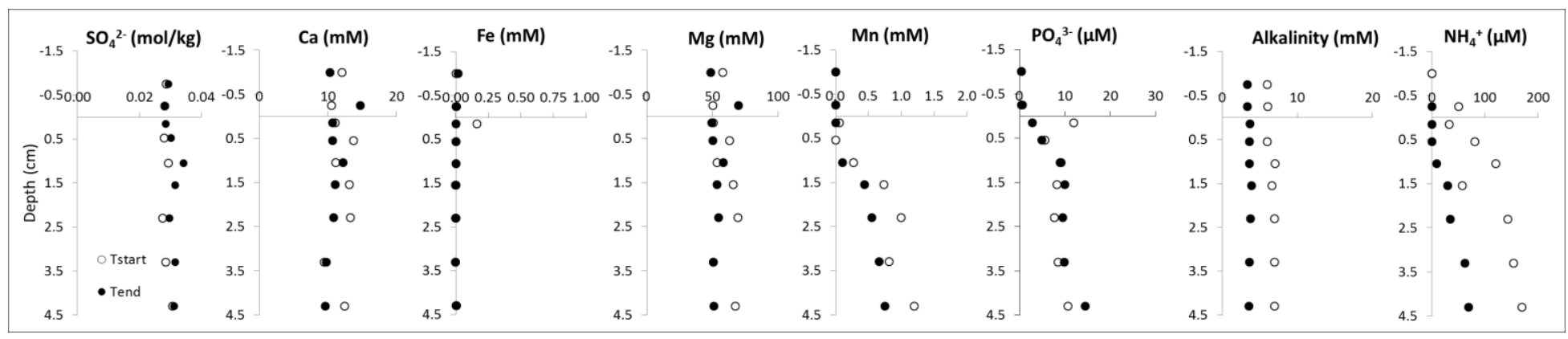

